\title{
Endocarditis due to Vancomycin Resistant Enterococcus faecium
}

Kleoniki Georgousi ${ }^{1}$, George Floros ${ }^{1}$, Ioannis Kachrimanidis ${ }^{1}$, Aikaterini Liodi ${ }^{1}$, Alexandros Tsantoulas $^{1}$, Maria Tzaki ${ }^{1}$, George Velisaris ${ }^{1}$, Olga Agora ${ }^{1}$, Sofia Tsiplakou ${ }^{1}$, Ioannis

Kyriazis $^{1}$, and Nikolaos Kafkas ${ }^{1}$

${ }^{1} \mathrm{KAT}$

March 18, 2021

\begin{abstract}
Endocarditis due to multidrug-resistant Enterococci and specifically multidrug-resistant Enterococcus faecium (E. faecium) is rare. Optimal antimicrobial therapy still remains undefined and a challeging issue. We report a case of native valve endocarditis due to vancomycin-resistant E. faecium (VRE) in a 65-years-old man with significant comorbid conditions.
\end{abstract}

\section{Endocarditis due to Vancomycin Resistant Enterococcus faecium}

Keywords: Infective endocarditis, Enterococcus faecium, Vancomycin-resistant

\section{Key Clinical Message}

This case report highlights the lack on published literature on multi-drug-resistantEnterococci infections and particularly onvancomycin-resistant E. faecium infective endocarditis in terms of accurate treatment without side effects.

Georgousi Kleoniki, M.D., Ph.D. ${ }^{1}$, Floros George,M.D. ${ }^{2}$,Kachrimanidis Ioannis, M.D. ${ }^{1}$, Liodi Aikaterini, M.D. ${ }^{1}$, Tsantoulas Alexandros,M.D. ${ }^{1}$, Tzaki Maria, M.D. ${ }^{1}$, Velisaris George, M.D. ${ }^{2}$,Agora Olga,M.D. ${ }^{2}$, Tsiplakou Sofia, M.D., Ph.D. ${ }^{3}$,Kyriazis Ioannis, M.D., Ph.D. ${ }^{1}$, Kafkas Nikolaos, M.D., Ph.D. ${ }^{2}$

${ }^{1}$ Internal Medicine Department, General Hospital of Attika "KAT", Kifissia, Greece

${ }^{2}$ Cardiology Department, General Hospital of Attica "KAT", Kifissia, Greece

${ }^{3}$ Microbiology Department, General Hospital of Attica "KAT", Kifissia, Greece

\section{Introduction}

Infective endocarditis (IE) is a potentially lethal disease and it has become more complex when it is caused by multidrug-resistant pathogens difficult to treat, such as multidrug-resistant Enterococci. Endocarditis due

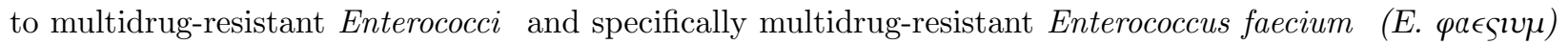
is rare as most of the reports of these strains are single case reports. VRE. faecium IE is an uncommon nosocomial infection that affect patients with significant comorbid conditions. Optimal antimicrobial therapy remains undefined and a challenging issue, but an attempt to identify bactericidal combination therapy should be sought.

We report a case of native valve endocarditis due to vancomycin-resistant E. faecium (VRE) that was paired with a clinical history of recent N-STEMI and previous surgical manipulations in the biliary tract.

\section{Case history}


A 65-year-old man presented to the emergency department with a $24 \mathrm{~h}$ history of repeated epigastric pain reflected to his back that was resolving with isosorbide dinitrate intake. Upon presentation in the ED, the patient denied fever, chills, cough, headache, sick contacts and recent travel.

His past medical history was significant for coronary artery disease, diabetes mellitus, hypertension and dislipidemia. His coronary artery disease had beguntwenty years ago. He had been submitted in a percutaneous coronary intervention angioplasty (PCI) in the LAD (2014) and a coronary artery bypass graft (CABG) for Left Main and three vessel disease (2017). Furthermore, he stated an episode of acute pancreatitis that led to cholecystectomy and insertion of biliary drainage catheter via percutaneous transhepatic cholangiography (PTC), which had been removed 2 months ago. The tissue biopsy for possible existence of cholangiocarcinomatosis was negative at that time, although a $4 \mathrm{~cm}$ mass was found anteriorly of the pancreatic head in abdomen CT. In addition, he stated a $18 \mathrm{kgr}$ body weight loss during the last 7 months and that he was on ciprofloxacin and metronidazole treatment the last week before admission, due to the previous episode of billiary occlusion.

A thorough physical examination was conducted and yielded the following results:

Vital signs: the patient was afebrile; pulse was 95 beats per minute (bpm); blood pressure 115/60mmHg (BP); oxygen saturation $99 \%$ (Sat02). There was no peripheral edema.

CVS: included S1, S2 with regular rhythm and diastolic murmur in the base.

Lungs: clear lungs with no wheezes or cough; mild dyspnea on exertion was present.

Abdomen: soft and non-tender; bowel sounds were present.

An ambulatory electrocardiogram (ECG) upon arrival exhibited sinus rhythm, mild tachycardia, presence of q wave on III and avF, and ST-wave segment depression with T-wave inversion on V3-V6. A transthoracic echocardiogram (TTE) was completed which revealed ejection fraction (EF): 50\%, mild dilated left atrium, the presence of a flail aortic valve leaflet and moderate to severe aortic regurgitation.

The current diagnosis on admission was Non ST Elevation Myocardial Infarction (N-STEMI) and the patient started taking metoprolol, rosuvastatin, aspirin, clopidogrel, ramipril, ezetimibe and glyceryl trinitrate. Coronary angiography was conducted which revealed coronary disease in three coronary arteries and well-functioning grafts. Left descending Coronary artery (LAD) had a severe stenosis $95 \%$ (culprit lesion) very distally beyond LIMA connection with LAD.

On day 9, the patient spiked a fever of $38,9^{\circ} \mathrm{C}$ with chills, blood cultures were taken and piperacillin/tazobactam was started. Physical examination revealed the same diastolic murmur. There were no peripheral stigmata of endocarditis like Osler's nodes, Janeway lesions or Roth's spots.

Laboratory results showed the following: white blood count (WBC) was $10.5 \times 10^{3} / \mathrm{uL}$ with $76,2 \%$ neutrophils (NEU); hemoglobin (Hb): 10,3g/dL; hematocrit (Hct): 31\%; platelets (PLT): 443x10 $/ \mathrm{uL}$; procalcitonin (PCT): 1,2ng/mL; C-reactive protein (CRP): 2,56mg/dL (0-0,5); creatinine (Cr): 1,1mg/dL, SGOT: $52 \mathrm{IU} / \mathrm{L}$; SGPT: $24 \mathrm{IU} / \mathrm{L}$; total bilirubin was $1,6 \mathrm{mg} / \mathrm{dL}$; and rheumatoid factor $(\mathrm{RF}): 563 \mathrm{IU} / \mathrm{ml}(<15)$. A new transthoracic echocardiogram showed the same flail aortic valve leaflet along with a second one, a finding compatible with infectious endocarditis.

The initial blood cultures revealed bacteremia due to Enterococcus faecium that was resistant to vancomycin (MIC, [?]256 mg/L), teicoplanin (MIC, [?]32mg/L), levofloxacin (MIC, [?]8mg/L), chloramphenicol (MIC, [?]16mg/L), imipenem (MIC, [?]16mg/L), gentamycin (HL)SYN-R, streptomycin (HL)SYN-R and susceptible to ceftaroline (MIC, 2mg/L), linezolid (MIC, 2mg/L), daptomycin (MIC, 1,5mg/L) and tigecycline (MIC, [?]0,12mg/L). In total, there had been taken four set of blood cultures, all of them positive to vancomycin-resistant E. faecium (VRE). As a consequence, piperacillin/tazobactam was discontinued and daptomycin (12mg/Kgr iv q.d), linezolid (600mg iv q12h) and tigecycline (150mg iv loading dose, $75 \mathrm{mg}$ iv $\mathrm{q} 12 \mathrm{~h})$ were started. 
After the new antibiotic regimen initiation, the patient was afebrile and the blood cultures became negative 4 days and 3 days respectively later. During the administration of antibiotics, the patient complained about headache, anorexia, nausea, dyspepsia, constipation and inability. Additionally, a gradual decrease in the procalcitonin (PCT):0,35ng/mL and C-reactive protein $(\mathrm{CRP}): 0,57 \mathrm{mg} / \mathrm{dL}$ levels were observed. However, we faced a significant reduction of platelets (PLT): 18x103/uL, due to myelotoxicity of linezolid. Hyperventilation and lactic acidosis (Lac): $12 \mathrm{mmol} / \mathrm{L}$ were further complications caused by the drug. Moreover, an increase in the liver enzymes (SGOT: 75IU/L, SGPT: 89IU/L) and international normalization ratio (INR): 1,92 (0,9-1,1) parallel with fibrinogen reduction to $1,0 \mathrm{~g} / \mathrm{L}(1,8-3,5)$, were observed propably due to tigecycline administration.

Having taken all the above into account, we decided to discontinue linezolid and tigecycline two weeks after their initiation and add ceftaroline (600mg iv q8h), to daptomycin.

In the same period, a transesophageal echocardiogram (TEE) showed the aortic valve having three (3) vegetations: on the right coronary cusp (RCC), on the left coronary cusp (LCC) and on the non-coronary cusp (NCC). It was noted moderate to severe aortic valve regurgitation. There was mild mitral valve regurgitation, free from any vegetation. There was no thrombus noted (Figure 1).

On day 28 of admission, the patient exhibited dyspnea, hypoxemia with peripheral edema. A chest X-ray showed mild pulmonary edema with small bilateral pleural effusions, for which furosemide was given.

On day 35, which was the ninth day after the discontinuation of linezolid and tigecycline, a mild rising in platelets (PLT) to 43,2x103/uL, a reduction of SGOT/SGPT in normal levels, and a fibrinogen and INR ratio normalization was noted. In the same evening, the patient's status became worse with chest tightness, dyspnea, and acute respiratory insufficiency. The arterial blood gas (ABG) test was: arterial blood pH: 7,34, bicarbonate: $9,7 \mathrm{meq} / \mathrm{L}$, partial pressure of oxygen: $67 \mathrm{mmHg}$, partial pressure of carbon dioxide: $18 \mathrm{mmHg}$ and lactic acid: $17,9 \mathrm{mmol} / \mathrm{L}$. The patient was intubated, and during the process he had an episode of cardiac arrest. A cardiopulmonary resuscitation (CPR) was followed by administration of adrenaline, atropine, and vasoconstrictors. The echocardiogram showed injection fraction (EF): $40 \%$, pulmonary artery systolic pressure (PASP): $60 \mathrm{mmHg}$, posterior wall immobility, and diffuse disimmobility. The patient was hemodynamically unstable and oligouric.

He was transmitted to ICU after anegative RNA-PCR test for COVID-19. He was set on continuous venovenous hemodiafiltration (CVVHDF). The patient's medical status complicated with hepatic ischemia, increase of INR ratio and the necessity of blood and plasma transfusions. In the midst of polyorganic insufficiency, the patient died 4 days later.

\section{Discussion}

Enterococci are gram-positive bacteria that are part of the normal gastrointestinal flora. They have become increasingly nosocomial pathogens resistant to many antimicrobials and they can survive in the environment for prolonged periods of time (1). Moreover, enterococci are considered the second leading cause of nosocomial infections (after staphylococci), including catheter-associated bacteremias. The more commonly described sources of enterococcal bacteremia are gastrointestinal and urinary tracts (with an important association with invasive procedures),indwelling catheters and anatomical abnormalities including the presence of malignant or inflammatory lesions.

The frequency of enterococcal bacteremia which results in IE varies widely. Risk factors for the development of IE in patients with enterococcal bacteremia include a history of pre-existing valvular heart disease, prosthetic valve, and older age with underlying diseases (2). The first case of vancomycin-resistant enterococci endocarditis that met the Duke criteria was reported in 1996 (3). Risk factors for the development VRE IE possibly are a history of livertransplantation, hemodialysis, prior long-term antibiotic use, increased severity of illness and corticosteroid treatment (4). An important issue in the epidemiology of enterococcal IE is the increase of infection caused by multidrug-resistant $E$. faecium .

The clinical presentation of enterococcal IE is usually subacute, with fever and the presence of a cardiac 
murmur as the most common findings in the physical examination. Classical signs of IE such as Osler's nodes or Roth's spots are less frequently found. The main complication of enterococcal IE is heart failure, which occurs in almost half of the patients and has an important impact on outcome.

Enterococci possess both intrinsic and acquired resistance properties. Their penicillin binding proteins allow them to resist beta-lactam antibiotics (5). Another problem correlates with the emergence and widespread dissemination of multidrug-resistant E. faecium isolates belonging to a hospital-associated (HA) genetic subclade that is responsible for most of the infections caused by this species (6). Genomic analyses have shown that the allelic variant of the gene encoding the penicillin-binding protein 5 (associated with highlevel resistance to ampicillin) and genes coding for aminoglycoside modifying enzymes are frequently found in E. faecium isolates belonging to this genetic lineage (clade A) (7). Tranditionally, vancomycin was the antibiotic of choice for treatment of ampicillin-resistant E. faecium infections; however E. faecium isolates are often resistant to vancomycin, making this antibiotic useless for the majority of E. faecium IE patients.

Unfortunatelly, there are no data available from randomized, controlled trials to determine optimal therapy. Treatment of enterococcal endocarditis necessitates a bactericidal synergistic combination of antimicrobials. Enterococci are considered to be resistant to vancomycin if MICs are $>4 \mathrm{mg} / \mathrm{L}$. For strains of enterococci resistant to $\beta$-lactams, vancomycin, or aminoglycosides, it is reasonable to test for susceptibility in vitro to daptomycin and linezolid according to the American Heart Association (AHA) guidelines (8). Linezolid is bacteriostatic in vitro against enterococci, whereas daptomycin is bactericidal in vitro in susceptible strains. Quinupristin-dalfopristin may be active in vitro but it is rarely used because of side effects, including intractable muscle pain. Tigecycline is active in vitro against some strains of multidrug-resistant enterococci, but there are minimal published data on its use.Furthermore, on the context of the current treatment guidelines there is an argument that VRE endocarditis is an indication for valve replacement on the basis of lack of effective antimicrobial therapy (9).

Because our patient was not a candidate for an operation due to underlying increased severity of illness, our goal was to identify a regimen that we believed would offer maximal bactericidal activity. We chose to treat him with daptomycin, linezolid and tigecycline according to the AHA guidelines (8). We were satisfied by the fact that the blood cultures became negative 7 days after the initiation of therapy.

Despite the fact that our patient was afrebrile 4 days after the begining of antibiotics, he exhibited common and even more challenging side effects due to linezolid andtigecycline intake. We decided to change them to daptomycin/ceftaroline according the available sensitivity test and the AHA guidelines: Combination therapy with daptomycin and ampicillin or ceftaroline may be considered, especially in patients with persistent bacteremia or enterococcal strains with high MIC's to daptomycin within the susceptible range (Class IIb;Level of Evidence C ) (8).

After the discontinuation of linezolid/tigecycline we observed a gradual normalization of the laboratory tests. However, our patient was suffering from critical illnesses. He had a recent episode of N-STEMI and previous hospitalizations with long courses of antibiotics and major surgical manipulations due to billiary tract obstruction. Although the hypothesis of possible cholangiocarcinomatosis had never been confirmed, we reasonably accept that our patient was a candidate for GI colonization and infection of vancomycin-resistant E. faecium . His already critical medical situation complicated with worsening of coronary artery disease, heart failure, pulmonary edema, pleural effusions and finally respiratory insufficiency.

This case report highlights the lack of published literature on multidrug-resistant enterococci infections and particularly the severity of vancomycin-resistant E. faecium IE in a critically ill patient. At the present time, there are no data available on the management of vancomycin-resistant E. faecium, and the optimal therapy of these species remains unknown.

Georgousi Kleoniki, M.D., Ph.D. ${ }^{1}$ X, Floros George, M.D. ${ }^{2}$,Kachrimanidis Ioannis, M.D. ${ }^{1}$, Liodi Aikaterini, M.D. ${ }^{1}$, Tsantoulas Alexandros,M.D. ${ }^{1}$, Tzaki Maria,M.D. ${ }^{1}$, Velisaris George,M.D. ${ }^{2}$, Agora Olga, M.D. ${ }^{2}$, Tsiplakou Sofia, M.D., Ph.D. ${ }^{3}$, Kyriazis Ioannis, M.D., Ph.D. ${ }^{1}$, Kafkas Nikolaos, M.D., Ph.D. ${ }^{2}$ 
XCorresonding author

Corresponding: Georgousi Kleoniki, M.D., Ph.D., Infectious Diseases Consultant, Internal Medicine Department, KAT General Hospital, Nikis Street 2, Kifissia, Attica, 14561, Greece. Tel:, +302132086338. Email:methexiskleri@gmail.com

\section{Author Contribution}

Georgousi Kleoniki: Designing the article, Collecting the data, Writing the manuscript, Editing the manuscript.

Floros George: Collecting the data.

Kachrimanidis Ioannis: Collecting the data.

Liodi Aikaterini: Collecting the data.

Tsantoulas Alexandros: Collecting the data.

Tzaki Maria: Collecting the data.

Velisaris George: Collecting the data.

Agora Olga: Collecting the data.

Tsiplakou Sofia: Microbiological analysis, Antibiogram interpretation.

Kyriazis Ioannis: Supervising the data.

Kafkas Nikolaos: Supervising the article, Collecting the data, Writing the manuscript.

\section{Acknowledgement}

We would like to thank Papaioannou Basiliki for microbiological analyses and the whole Cardiology and Internal Medicine Department for the support.

References:

(1). Stevens MP, Edmond MB. Endocarditis due to vancomycin-resistant Enterococci: Case report and review of the literature. Clin Infect Dis. 2005;41:1134-1142.

(2). Megran DW. Enterococcal endocarditis. Clin Infect Dis. 1992;15(1):63-71.

(3). Bishara J, Sagie A, Samra Z, Pitlik S. Polymicrobial endocarditis caused by methicillin-resistant Staphylococcus aureus and glycopeptide-resistantenterococci. Eur J ClinMicrobiol Infect Dis. 1999;18:674-5.

(4). Gold H: Vancomycin-resistant enterococci: Mechanisms and clinical observations. Clin Infect Dis. 2001;33(2):210-19.

(5). Lebreton F, van Schaik W, McGuire AM, Godfrey P, Griggs A, Mazumdar V, et al. Emergence of epidemic multidrug-resistant Enterococcus faecium from animal and commensal strains. MBio.2013;4.

(6). Galloway-Pena J, Roh JH, Latorre M, Qin X, Murray BE. Genomic and SNP analyses demonstrate a distant separation of the hospital and community-associated cladesof Enterococcus faecium . PLoS One. 2012;7(1):e30187.

(7). Galloway-Pena JR, Rice LB, Murray BE. Analyses of PBP5 of early U.S. isolates of Enterococcus faecium: sequence variation alone does not explain increasing ampicillin resistance over time. Antimicrob Agents Chemother. 2011;55:3272-7.

(8). Baddour LM, Wilson WR, Bayer AS, Fowler VG, Tleyjeh IM, Rybak MJ, et al. Infective endocarditis in adults: diagnosis, antimicrobial Therapy, and management of complications. Circulation AHA. 2015;132:1435-1486. 
(9). Olaison L, Pettersson G. Current best practices and guidelines: indications for surgical intervention in infective endocarditis. Infect Dis Clin North Am. 2002;16:453-75.

\section{FIGURES}

Fig. 1. TEE showed the aortic valve having three (3) vegetations

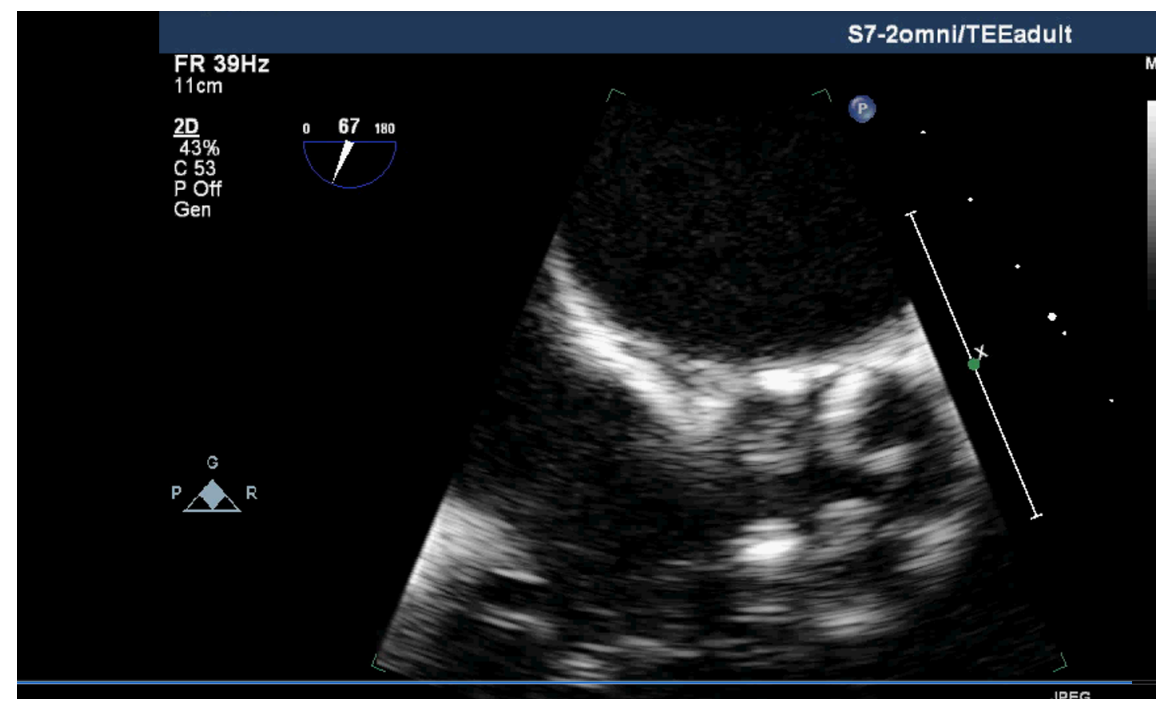

